\title{
El derecho a la igualdad de género en el ámbito educativo en el contexto de la pandemia covid-19
}

\author{
The right to equality in education in the context of the covid-19 pandemic
}

O direito à igualdade na educação no contexto da covid-19 pandemia

\section{ARTÍCULO DE REVISIÓN}

\author{
Mercedes Navarro Cejas \\ mercedes.navarro@utm.edu.ec \\ https://orcid.org/0000-0003-4377-7250
}

\author{
Hipatia Delgado Demera \\ hipatia.delgado@utm.edu.ec \\ https://orcid.org/0000-0002-5815-8981
}

Universidad Técnica de Manabí, Manabí-Ecuador

Recibido 08 de enero 2021 | Arbitrado y aceptado 15 de febrero 2021| Publicado en 01 abril 2021

\section{RESUMEN}

El propósito de artículo es mostrar un análisis en el contexto jurídico y social en el que se desenvuelve la igualdad de género en la educación a raíz de la pandemia COVID 19. Al respecto se pudo evidenciar que las medidas tomadas antes de la crisis sanitaria y los logros que se habían alcanzado se ven comprometidos en el contexto de pandemia que atraviesa el mundo en la actualidad. El estudio se llevó a cabo bajo una investigación documental con un análisis de información cualitativa, con diseño no experimental, longitudinal $\mathrm{y}$ con respecto a su alcance es de carácter exploratorio. Se llegó a la conclusión que las mujeres y las niñas en la educación están expuestas a sensibles repercusiones tras la pandemia que pueden comprometer su empoderamiento en este aspecto.

Palabras clave: Igualdad de género; Ecuador; mujer; educación; pandemia

\section{ABSTRACT}

The purpose of the article is to show an analysis in the legal and social context in which gender equality unfolds in education as a result of the COVID 19 pandemic. In this regard, it was possible to show that the measures taken before the health crisis and the achievements that had been achieved are compromised in the context of the pandemic that the world is currently experiencing. The study was carried out under a documentary investigation with an analysis of qualitative information, with a nonexperimental, longitudinal design and with respect to its scope it is exploratory in nature. It was concluded that women and girls in education are exposed to sensitive repercussions after the pandemic that can compromise their empowerment in this regard.

Keywords: Gender equality; Ecuador; woman; education; pandemic

\section{RESUMO}

O objetivo do artigo é apresentar uma análise no contexto jurídico e social em que a igualdade de gênero se desdobra na educação a partir da pandemia COVID 19. Nesse sentido, foi possível evidenciar que as medidas tomadas antes da crise sanitária e as conquistas alcançadas estão comprometidas no contexto da pandemia que o mundo vive atualmente. 0 estudo foi realizado no âmbito de uma investigação documental com análise de informação qualitativa, com um desenho não experimental, longitudinal e quanto ao seu âmbito é de natureza exploratória. Concluiu-se que mulheres e meninas na educação estão expostas a repercussões sensíveis após a pandemia que podem comprometer seu empoderamento nesse sentido.

Palavras-chave: Igualdade de gênero; Equador; mulher; Educação; pandemia 


\section{INTRODUCCIÓN}

Se entiende por igualdad de género como un elemento esencial para la construcción de sociedades que permite el desenvolvimiento de todos los grupos vulnerables, entre ellos, las mujeres (Herrera, 2017). En tal sentido, Ferrajoli (2020) indica que cuando se trata de la igualdad se deben considerar entonces las diferencias sustanciales de todas las personas cuestión que constituye su identidad en el contexto jurídico que representa a las sociedades modernas. Al lado de la igualdad se debe hacer referencia también a las formas de discriminación.

Con respecto a la discriminación algunos autores como Pons (2015) y Orbegoso (2016) resaltan la importancia de conquistar la igualdad material que no es más que la materialización de las políticas de igualdad a los fines de que se apliquen y cumplan en la esfera práctica de aplicación de la norma. En tal sentido, sea como sea que se apliquen estas medidas debe tomarse en cuenta el principio de proporcionalidad entre las políticas públicas que se tomen y la finalidad deseada, es decir, que exista una proporción entre la finalidad que tiene la norma y la medida jurídica que se ha tomado en torno a ella. Por lo tanto, debe haber una relación razonable entre ambas.

En este aspecto, habría entonces que referirse de forma concreta a la igualdad de género en la educación, sin embargo, a pesar de los esfuerzos que se han realizado al respecto, lo cierto es que históricamente la incorporación de la mujer en cualquier nivel de enseñanza ha supuesto la modificación de las normas jurídicas a los fines de incorporar componentes legislativos que permitan que ellas formen parte, precisamente, de estos espacios (Navarro, 2017). Tal y como lo indican Navarro, Serpa y Cejas (2018) en el mundo, incluso con las nuevas políticas que se manejan al respecto, sigue existiendo una considerable brecha entre las normas jurídicas y las sensibles realidades que viven las mujeres y las niñas en todos los ámbitos en los que se desarrollan, especialmente en el ámbito educativo.

Algunos autores, que han analizado estos temas a nivel mundial hacen referencia a que es una realidad que se propaga en todo el mundo. A manera de ejemplo, podría hablarse de varios contextos entre ellos África (González y Ledesma, 2016), Europa (Ramírez y Matarranz, 2015) o América Latina (Céspedes y Robles, 2016). Ahora bien, algo en común que tienen estos autores, es que todos reconocen que las políticas creadas sobre la igualdad de género y la efectividad que pueden llegar a tener nacen $y$ se sustentan en la educación de las personas, en la forma en la que se proyectan los roles de género en los centros educativos, en la forma como se llevan a cabo acciones concretas en este aspecto, cuestión que también va a contribuir de forma significativa en la consecución de los fines de las políticas de acción positiva que mejoren las condiciones de vida de las mujeres y las niñas, a través de la propagación de medidas que promuevan la igualdad entre los colectivos sociales en la educación.

Sin embargo, a pesar de los innumerables esfuerzos que se han llevado a cabo, la sociedad atraviesa por realidad palpable como lo es la pandemia COVID 19, que ha llevado a la modificación de todos los escenarios legislativos a los fines de intentar contener la extensa y rápida propagación del virus, cuyos síntomas pueden llegar a ser mortales (Hermi y García, 2020). 
El trabajo que el presente trabajo tiene como objetivo analizar la problemática relacionada con la igualdad y no discriminación en el ámbito educativo de la mujer contextualizada dentro de la pandemia COVID 19.

\section{METODOLOGÍA}

La modalidad paradigmática utilizada en este estudio responde a una metodología cualitativa. Al respecto, Gómez et. al. (2017) indica que trata de un estudio basado mayoritariamente en valoraciones lo que deja en descubierto que no fueron manipuladas variables y más bien estamos en presencia de la utilización del método empírico de revisión documental. Por ende, se realizó una revisión bibliográfica actualizada que permitió la construcción de un marco teórico referencial en el que se tuvo en cuenta el punto de partida la siguiente interrogante: ¿Cómo se ha desarrollado el derecho a la igualdad en el ámbito educativo dentro del contexto de la pandemia COVID 19?

La respuesta a esa interrogante se desarrolla suficientemente en esta investigación. Para ello, se hizo uso de un diseño longitudinal que permitió estudiar el desarrollo en el tiempo de un fenómeno, en este caso, la igualdad en el ámbito educativo antes y durante la pandemia COVID 19. A su vez, se enmarcó en una investigación no experimental, lo que quiere decir que las variables no fueron manipuladas; fue de carácter etnográfico porque permitió la descripción e interpretación profunda de un fenómeno donde se estudian categorías, temas, problemas y patrones referidos a sucesos o culturas (Gómez, et al., 2017).

Esta investigación es de alcance descriptivo, cuestión que permite analizar cómo es y cómo se desarrolla y manifiesta un fenómeno en el tiempo (Gómez, et. al., 2017, p.50). Adicionalmente se hizo uso del método analítico- sintético e inductivo- deductivo los cuales permitieron realizar un análisis general de la investigación y sintetizar en sus partes más importantes al respecto, a su vez se fue de lo general a o particular y de lo particular a lo general.

\section{DESARROLLO Y DISCUSIÓN}

A los fines de poder contextualizar la realidad que existe dentro del ámbito educativo, se hace necesario hacer una primera aproximación sobre los esfuerzos realizados a nivel internacional al respecto. En una primera aproximación la educación alcanza su ulterior formulación dentro del Programa Mundial para la Educación en Derechos Humanos de Naciones Unidas, A/HRC/15/28, del 10 de diciembre de 2004 cuyo Plan de Acción en su segunda etapa se señala dentro de sus finalidades: "Promover la comprensión, la tolerancia, la igualdad entre los géneros y la amistad entre todas las naciones, los pueblos indígenas $\mathrm{y}$ las minorías" (ONU, 2012, p. 13). Sin embargo, a pesar de este avance inicial, uno de los más significativos logros con respecto al tema de análisis es que la igualdad de género en la educación se incluyó en el objetivo número 3 de los "Objetivos del Desarrollo del Milenio" vigentes hasta el 2015 que era "Promover la igualdad de género y el empoderamiento de la mujer".

Dentro de este objetivo existía también la meta 3.A. que trataba sobre las desigualdades entre los sexos en la enseñanza primaria y secundaria. Al respecto, el informe final de dichos objetivos indicaba que para el año 2015 si bien eran muchas más niñas las que asistían a la escuela que hace 15 años aun existían importantes debilidades dentro de 
los sistemas educativos además de otros factores que impedían la consecución de la igualdad de género en el ámbito educativo por este motivo se incluye dentro del objetivo número 4 "Garantizar una enseñanza inclusiva y equitativa de calidad y promover oportunidades de aprendizaje permanente para todos" cuya meta 4.1 es que "De aquí a 2030, asegurar que todas las niñas y todos los niños terminen la enseñanza primaria $\mathrm{y}$ secundaria, que ha de ser gratuita, equitativa $\mathrm{y}$ de calidad y producir resultados de aprendizaje pertinentes y efectivos" de los Objetivos del Desarrollo Sostenible, vigentes hasta el 2030 (ONU, 2021).

Ahora bien, con respecto al cumplimiento de este objetivo para el 2019 existe un informe de la ONU sobre el cumplimiento de los ODS antes de la pandemia COVID 19. Dicho informe, al respecto de nuestro tema de estudios indica que en la actualidad "millones de niños siguen sin asistir a la escuela y no todos los que asisten aprenden. Más de la mitad de todos los niños y adolescentes del mundo no están alcanzando los niveles mínimos de competencia en lectura y matemáticas" (ONU, 2019, p. 30). Esto, aun cuando no lo parezca repercute en las economías de los países al tener personas menos preparadas para hacer frente al competitivo mercado laboral.

A nivel mundial, a pesar de que las niñas estadísticamente tienen mejores capacidades de lograr un nivel mínimo de competencia en lectura ( ya que de 100 niños en lograr un nivel mínimo de competencia en lectura 105 niñas lo consiguen) las mujeres siguen afrontando en la educación persistentes desigualdades que les impiden finalizar sus estudios, siendo una de ellas que mantienen las labores y cargas del hogar desde temprana edad, por lo que aún muchas no asisten a la escuela o no terminan sus estudios. Solo a nivel mundial por cada 100 niños, son 118 las niñas que no asisten a la escuela y de hecho, 750 millones de adultos todavía no saben leer ni escribir y dos tercios de esos adultos son mujeres (ONU, 2019).

Sin embargo a pesar de esas realidades, bien indica la ONU (2019) que hoy el mundo es un lugar mejor para las mujeres y las niñas. Algunos datos estadísticos mencionados por la Organización, indican que actualmente "son menos las niñas forzadas a contraer matrimonio a temprana edad, más mujeres están sirviendo en el parlamento y en cargos de liderazgo, y se están reformando las leyes para promover la igualdad de género" (ONU, 2019, p. 32). En materia educativa, para poder analizar esta realidad con un mayor índice de profundidad hace falta hacer mención a otros factores que son de importante relevancia. Uno de ellos es que, por ejemplo aun en la actualidad las mujeres dedican por lo menos el triple de tiempo que los hombres al trabajo doméstico no remunerado, según lo indican los datos de 90 países a nivel mundial.

Ahora bien, este factor, que parece ser aislado, es realmente muy importante. Lo cierto es que el trabajo doméstico incluye actividades como el cuidado de niños y ancianos, tareas de limpieza etc. Además la concepción social de que deben ser más mujeres quienes ejerzan estas funciones las dejan expuestas a un rol que resta tiempo para que se dediquen a otras actividades que podrían garantizar su empoderamiento económico como es el caso de la educación. Este es el motivo principal por el que muchas mujeres no empiezan su educación o no la terminan y en el mercado laboral no suelen ser tan competitivas como los hombres. (ONU, 2019). 
Ante esta realidad palpable, el mundo enfrenta una crisis sanitaria sin precedentes que ha comprometido considerablemente los espacios para las políticas públicas. En el informe antes mencionado la ONU había realizado un estudio sobre las formas en las que se venía desarrollando el cumplimiento de los ODS hasta antes de la pandemia pero en la actualidad habría que analizar las sensible realidad del mundo mientras se desarrolla la crisis sanitaria. Al tratarse de una realidad que se encuentra en desarrollo solo podría hablarse de proyecciones. En tal sentido se hace necesario hacer referencia al informe " Educación, Género y COVID-19" del Fondo de las Naciones Unidas para la Infancia (UNICEF 2021) y al "Informe de políticas: La educación durante la COVID-19 y después de ella" (ONU, 2020).

En tal informe, más allá de las realidades que hemos analizado con anterioridad, se mencionan otras que también son importantes, por ejemplo el hecho de que las niñas que no asisten a las escuelas tienen una mayor probabilidad de ser obligadas a casarse o unirse de alguna forma, a un hombre. Adicionalmente, ausentarse de las aulas pueden conllevar a alimentar la idea de que las mujeres deben mantener la función reproductiva dentro de la sociedad. Esto debido a que si no estudian o se preparan no tienen la capacidad de comprender el mundo desde una perspectiva distinta. Esta realidad se mantiene en América Latina de una forma palpable: una prueba de ello es que por lo menos en países como Ecuador el 49,3\% de los nacimientos son de madres adolescentes, en Perú el 13\% de las niñas en edad escolar son madres antes de la mayoría de edad y en
Bolivia esta cifra aumenta al $34 \%$ para las mujeres que declararon alguna vez estar embarazadas (UNICEF, 2021).

Ahora bien, dentro del contexto de la pandemia, las estadísticas muestran realidades alarmantes. Una de ellas es la violencia sexual presente en los hogares a nivel mundial y que se traduce como un indicador que incide directamente en el rendimiento escolar de los niños y niñas sin distinción de sexo. A propósito del comentario anterior, a pesar de que el efecto repercute de forma negativa para cualquier sexo lo cierto es que las mujeres están mucho más expuestas a este tipo de violencia dentro del contexto de la crisis sanitaria. Prueba de ello es que por ejemplo en Colombia una mujer es víctima de violencia sexual cada 23 minutos y en el confinamiento por lo menos 51 niñas sufren violencia sexual diariamente (UNICEF, 2021).

Otra realidad palpable es el manejo de la higiene menstrual. Dentro del contexto de la crisis sanitaria que también es una crisis económica en incremento de los costos de la vida y de los productos menstruales podría obligar a las mujeres y a las niñas a decidir entre acceder a estos artículos o a la escuela y otros servicios (UNICEF, 2021). En tal sentido el tiempo que podrán dedicarle las niñas y adolescentes a sus estudios se está viendo afectado no solamente por el cierre de las escuelas sino también por todos los factores que aquí se mencionan. En este contexto, la UNICEF (2021) propone ciertas recomendaciones, (Figura 1) las cuales se resumen de forma concreta en el siguiente recuadro, donde se han mencionado las más importantes: 


Se deberán promover alianzas
para abordar temas
transversales de salud,
educación y protección social
que puedan tener un impacto en
la continuidad del aprendizaje y
el regreso a la escuela.

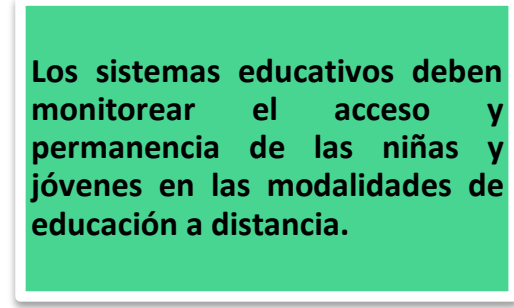

Los sistemas educativos deben monitorear el acceso y permanencia de las niñas y jóvenes en las modalidades de educación a distancia.
Se deben tomar medidas para hacer frente a la brecha digital basada en género, promoviendo las habilidades y competencias que les permitan a las niñas navegar en línea de forma segura

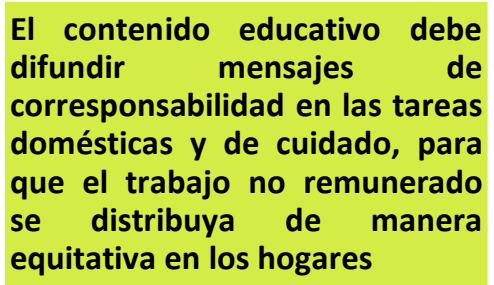

En el contexto de la crisis sanitaria las respuestas que den los centros educativos deben tomar en cuenta las dimensiones de género considerando las necesidades de mujeres y niñas como grupos vulnerables, de lo contrario estarían en riesgo 20 años de medidas para promover la igualdad en la educación.

Figura 1. Recomendaciones basadas en la Unicef. Fuente: Elaboración propia a partir de UNICEF (2021).

Ahora bien, con respecto al "Informe de políticas: La educación durante la COVID-19 y después de ella" (ONU, 2020). También es preciso hacer algunas consideraciones importantes. Tal documento, al plantear el contexto de la pandemia indica que cerca de 23,8 millones de niños y jóvenes por lo menos en etapa escolar podrían abandonar la escuela o no tener recursos económicos para seguir en ella debido a las consecuencias manifiestas de la pandemia eso sin mencionar que el cierre de las instituciones educativas ha generado otros efectos para los niños como la paralización de prestación de servicios esenciales de comidas para algunos de ellos que tienen realidades palpables. En el poco tiempo que tiene la pandemia por lo menos 395 millones de niños han sido afectados por esto, lo que provocó un aumento del hambre y las carencias alimentarias de los más desfavorecidos (ONU, 2020).

La crisis económica podría también comprometer el mantenimiento de las escuelas como centros educativos y sus recursos para generaciones venideras. La ONU (2020) indica que las proyecciones para los países de ingreso bajo y mediano bajo, por ejemplo, alcanzan una suma de 148.000 millones de dólares estadounidenses anuales, una suma que podría incrementarse ahora en hasta un tercio por lo que la crisis económica no va a tener buenas repercusiones para las escuelas. Otra realidad es que los planes de reapertura de los centros educativos (Figura 2) no son concisos con respecto a las fechas en las podrían reabrirse (ONU, 2021). En el siguiente recuadro, se describe una proyección al respecto: 


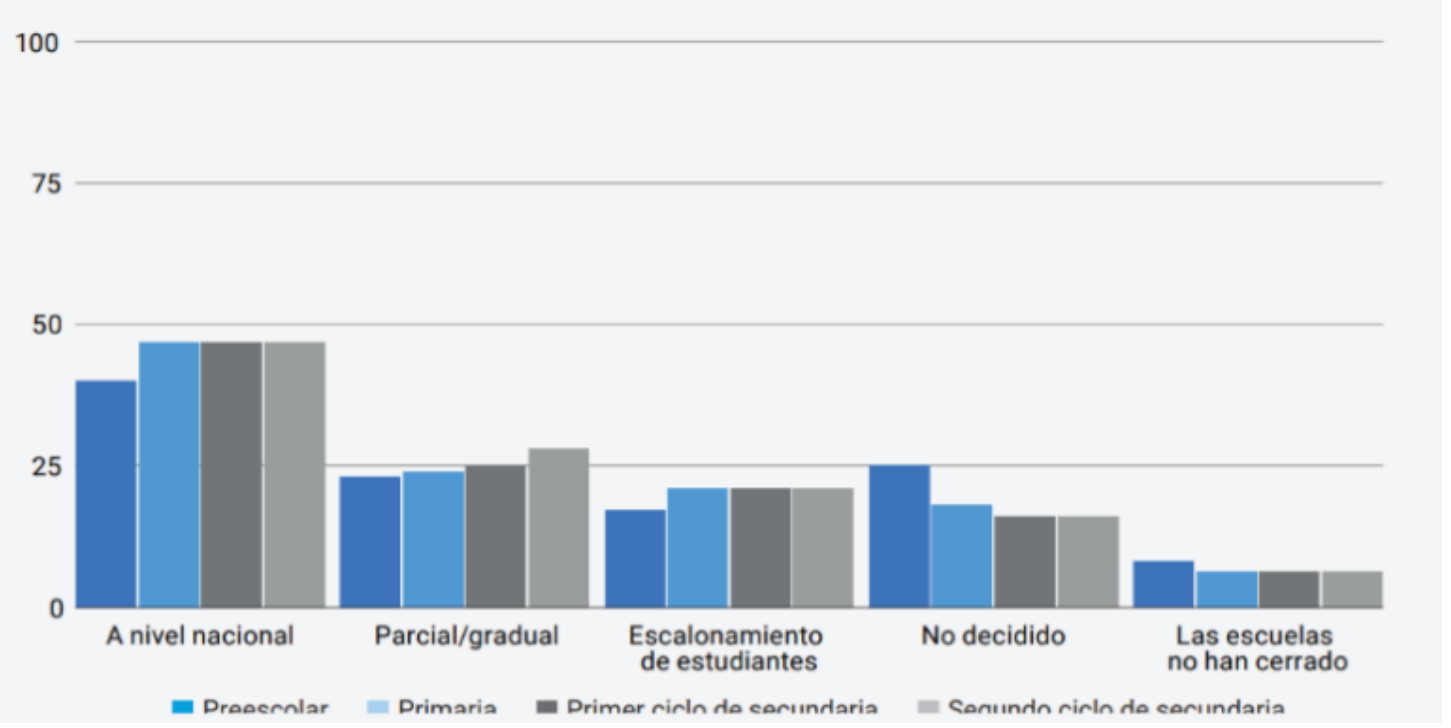

Figura 2. Planes de reapertura de escuelas por nivel. Fuente: ONU 2021.

\section{CONCLUSIONES}

Los avances que se han visto manifestados en los distintos ordenamientos jurídicos sobre la igualdad de género en la educación hasta antes de la pandemia COVID19 son de interés para la comunidad científica que sigue de cerca estas medidas y las estadísticas que sostienen su efectividad. Del estudio realizado en esta investigación puede verse otra realidad sensible y es que a pesar de esas formas legislativas y políticas públicas implementadas estamos en presencia de situaciones que se mantienen en la actualidad. La desigualdad sexual es una consecuencia de la desigual distribución de las funciones y los roles de ambos sexos cuestión que genera disparidades de género y que impide la consecución de la igualdad.

La educación es uno de los pilares fundamentales para hacer frente a las formas de discriminación ya que es el espacio donde se forma a las personas para la sociedad. Todo esto conlleva también a hacer referencia a la crisis sanitaria que afronta nuestro mundo la cual ha generado importantes repercusiones en todos los ámbitos, en especial en el ámbito económico y educativo. La llegada de la teleducación o de la educación ha distancia ha supuesto la reinvención de los sistemas de enseñanza pero ha dejado ver las sensibles situaciones que viven muchas mujeres y niñas a nivel mundial.

El aumento de los índices de violencia contra las mujeres, la carga doméstica, y los pocos recursos económicos con los que cuentan algunas de ellas ha comprometido su desempeño educativo en muchos casos en su totalidad. Es importante reforzar la idea de que existan mejores condiciones para estas personas por lo que las medidas que hagan frente a la crisis sanitaria deben hacerlo también en el contexto de la inclusión de criterios sensibles al género.

En forma de propuesta, los centros educativos deben considerar estas realidades y realizar verdaderos seguimientos a los estudiantes en especial a los que forman parte de estos grupos vulnerables. Es importante 
que las escuelas sigan contando con recursos económicos para su reapertura, sobre todo con planes de alimentación escolar incluso durante esta época en la que se están cerradas en su mayoría con comidas diarias entregadas a domicilio y raciones mensuales para llevar. Sin embargo, en el contexto de la pandemia, para lograr la igualdad de género se requerirán medidas audaces y sostenibles que consideren y tomen en cuenta todos los impedimentos estructurales y las causas fundamentales de la discriminación contra la mujer. Esto también debe complementarse con leyes y normativas que ayuden a la promoción de medidas de igualdad de género incluso en la crisis sanitaria, que permitan que además estas políticas publican cuenten con un respaldo adecuado de recursos que vayan encaminados a hacer cumplir responsablemente estos compromisos.

\section{REFERENCIAS}

Céspedes, C. y Robles, C. (2016). Niñas y adolescentes en América Latina y el Caribe. Deudas de igualdad. Santiago: CEPAL. Recuperado el 15 de Mayo del 2021

http://repositorio.cepal.org/bitstream/ha ndle/11362/40180/S1600427_es.pdf?seq uence $=1$

Ferrajoli, L. (2020). Manifiesto por la igualdad. Editorial Trotta. Madrid

Fondo de las Naciones Unidas Para La Infancia "Unicef" (2021). "Educación, Género y COVID-19". Recuperado el 15 de mayo del 2021 de https://lac.unwomen.org/es/digiteca/pu blicaciones/2020/06/educacion-generoy-covid-19

Gómez C., Álvarez G., Romero A., Castro F., Vega V., Comas R. Y Velázquez M. (2017). La investigación científica y las formas de titulación: aspectos conceptuales $y$ prácticos. Editorial Jurídica del Ecuador. Ecuador
González, M. I., y Ledesma, M. (2016). Mujeres africanas y educación superior: políticas educativas favorables a la igualdad de género en África subsahariana. Journal of Supranationl Policies of Education, (3), 156-183

Hermi, M., y Blas- Garcia M. (2020). "El COVID en España y sus primeras consecuencias" Revista brasileira de geografía económica. Revista Brasileña de Geografía Económica. Volumen 17. pp 1-20

Navarro M, Serpa G y Cejas M. (2018). El Derecho A La Igualdad En El Ámbito Educativo: Una Perspectiva Moderna Para La Inclusión De La Mujer. Revista de Derecho Ius Humani. Vol. 7. 89-104. DOI: https://doi.org/10.31207/ih.v7i0.171

Navarro M. (2017) La igualdad de género como derecho humano: un análisis desde la perspectiva del derecho internacional en el ámbito laboral. Editorial Jurídica del Ecuador. Quito. 2017. Página 35

Organización de las Naciones Unidas ONU (2020) "Informe De Políticas: La Educación Durante La COVID-19 Y Después De Ella" Recuperado el 15 de Mayo del 2021 de: https://www.un.org/sites/un2.un.org/file s/policy_brief_-_education_during_covid19_and_beyond_spanish.pdf

Organización de las Naciones Unidas ONU (2012). Plan de Acción. Programa Mundial para la Educación en Derechos Humanos, segunda etapa. Recuperado el 15 de Mayo del 2021 de https://documents-ddsny.un.org/doc/UNDOC/GEN/G10/151/51 /PDF/G1015151.pdf?OpenElement (visitado el 10 de agosto de 2017).

Organización de las Naciones Unidas ONU (2019). Informe de los Objetivos de Desarrollo Sostenible. Ginebra: Naciones Unidas. Recuperado el 15 de Mayo del 2021

de https://unstats.un.org/sdgs/report/2019 /The-Sustainable-Development-GoalsReport-2019_Spanish.pdf 
Orbegoso M. (2016). "La igualdad material como fin del Estado Social". Revista de estudiantes Ita Ius Esto (1), 1-10

Pons E. (2015). Igualdad y no discriminación por razón de sexo: nociones jurídicas básicas. Análisis Feminista del Derecho: teorías, igualdad interculturalidad y violencia de género (coordinación de
SÁNCHEZ A. y PUMAR BELTRÁN N.). Publicaciones de la Universidad de Barcelona. España

Ramírez, E., y Matarranz, M. (2015). Igualdad de género en educación. El caso de la Unión Europea. Journal of Supranationl Policies of Education, (3), 114-136 